\title{
Magnetic resonance images of left ventricular pseudoaneurysm
}

\author{
R N Chakraborty, A A Nicholson, M F Alamgir
}

A 63 year old woman presented in March 1997 with a three month history of shortness of breath while lying flat and in the left lateral position. She had a history of ischaemic heart disease and had coronary artery bypass surgery five years ago. In August 1995 she had an inferior wall myocardial infarction with postinfarction angina, which responded to medical treatment. Echocardiographic window was poor because of obesity; however, there appeared to be a large echo free space postero-inferior to the left ventricle leading to suspicion of left ventricular pseudoaneurysm. Magnetic resonance images of the heart performed in a 0.5 Tesla GE Vectra (IGF Medical, Milwaukee, Wisconsin, USA) magnetic resonance scanner were consistent with this diagnosis (figs 1-4).

The clinical presentation did not suggest a diagnosis of left ventricular pseudoaneurysm. Chest radiography showed cardiomegaly with left sided pleural effusion. In view of her previous heart attack and coronary artery bypass operation transoesophageal echocardiography was performed followed by a transthoracic echocardiography. The patient was considerably breathless while manoeuvring the probe. However, from a limited study there was a very strong suspicion of left ventricular pseudoaneurysm. After stabilising the patient we performed cardiac catheterisation and angiography. Left ventriculography showed that

Department of

Cardiology, Hull Royal Infirmary, Anlaby Road, Hull HU3 2JZ, UK

R N Chakraborty M F Alamgir

Department of Radiology, Hull Royal Infirmary

A A Nicholson

Correspondence to: Dr Chakraborty

Accepted for publication 6 January 1998

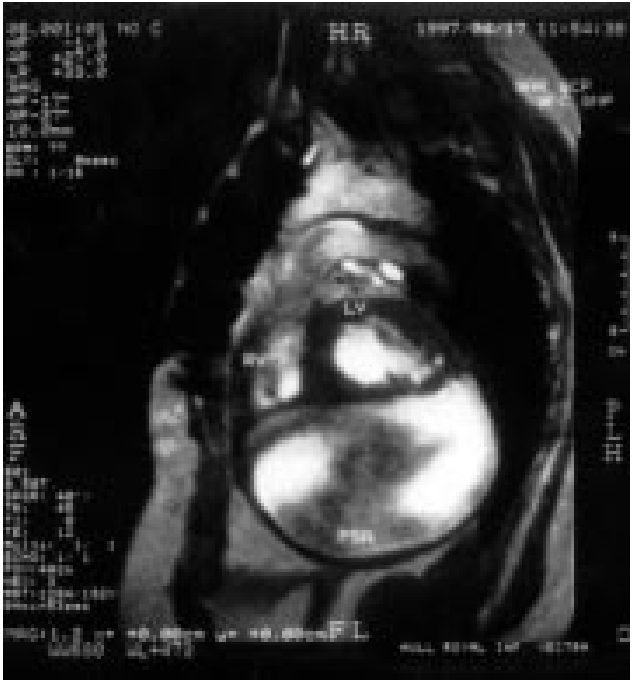

Figure $1 T_{2}$ weighted spoiled grass image from a cine loop sequence. A large thin wall, sac-like structure (PSA) is seen below the base of the left ventricle $(L V)$. contrast filled the pseudoaneurysm, but it was diluted in a huge sac.

The patient had open heart surgery, which revealed dense adhesions around the heart.

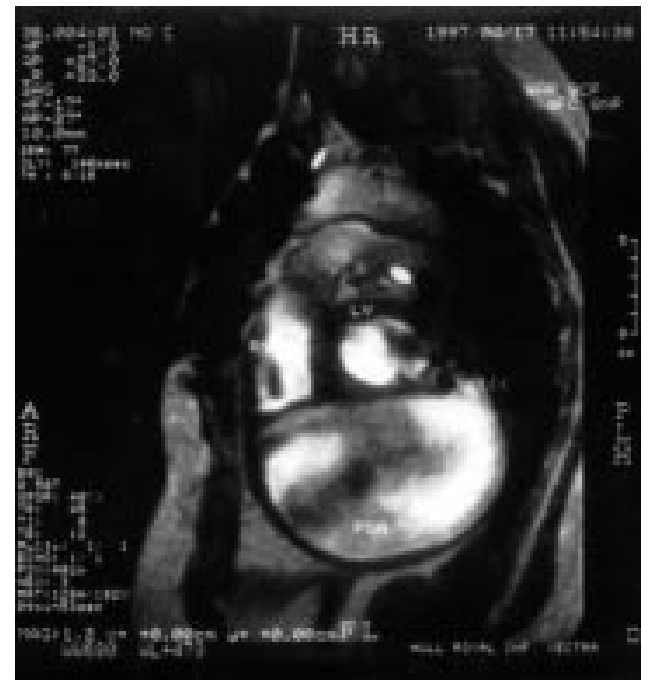

Figure 2 In midsystole a communication between the postero-basal area of the left ventricle (LV) and the thin walled, sac-like structure (PSA) is seen (arrowheads). The magnetic resonance image of this thin walled structure appears to be consistent with left ventricular pseudoaneurysm.

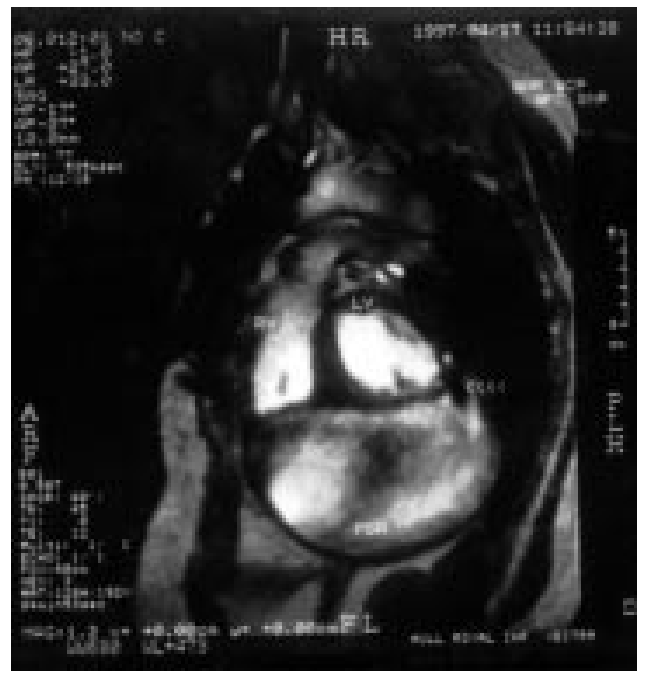

Figure 3 By late systole a flow void is seen (arrowheads) representing a jet of blood from the left ventricle into the pseudoaneurysm. Blood could be seen swirling around inside the pseudoaneurysm. 


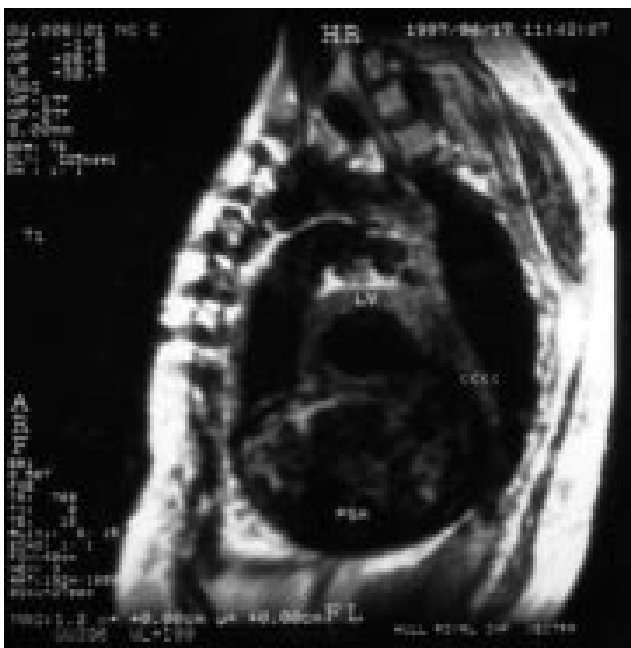

Figure $4 \quad T_{1}$ weighted gated image at the same level demonstrated the communication between the left ventricle and the pseudoaneurysm (arrowheads). Signal from inside the pseudoaneurysm indicates swirling of blood around with possible thrombus.
The pseudoaneurysm was very large and adherent to the left ventricle, extending over the right ventricle and right atrium. It was not possible to dissect it off the right atrium. After a long dissection the right atrium was split open exposing the tricuspid valve. The pseudoaneurysm was partially resected from the right ventricle. Its connection with the left ventricular wall was identified and the hole was closed. The pseudoaneurysm sac was wrapped postero-inferiorly around the heart encroaching on the right ventricle and the right atrium. The right atrium was densely adherent to the aneurysm wall posteriorly.

After dissection the right atrium was reconstructed. There were several areas of bleeding from the right atrium, which were secured. As it was dissected out from the ventricular walls there was profuse bleeding from the heart surface. Haemodynamically the patient became very unstable, her condition gradually deteriorated and she died.

\title{
SHORT CASES IN CARDIOLOGY
}

\section{Coronary stenting for symptomatic myocardial bridging}

\author{
A Bayés, V Martí, J M Augé
}

A 65 year old man was referred because of an episode of syncope preceded by palpitations. $\mathrm{He}$ had a four month history of chest pain and palpitations unrelated to exercise. On admission, ECG showed sinus rhythm without repolarisation abnormalities. Echocardiography Doppler evaluation was normal. In an exercise thallium-201 test the patient achieved $76 \%$ of the predicted maximal heart rate with a 4.6 Mets workload. A $2 \mathrm{~mm}$ ST segment depression was observed in the anterolateral leads without chest pain. A thallium-201 scan showed myocardial perfusion abnormalities involving the anterior segment. The Holter recording revealed two runs of non-sustained monomorphic ventricular tachycardia (VT) at 160 beats $/ \mathrm{min}$, which the patient felt as palpitations but without angina. Programmed stimulation with ventricular pacing and extrastimuli did not induce VT. Left coronary angiography showed a significant systolic milking effect in the second portion of the left anterior descending coronary artery (fig 1A), which appeared normal during diastole (fig 1B). There was no associated arteriosclerotic coronary disease. The patient was discharged and prescribed atenolol.
Six weeks later, the patient was readmitted for angina without arrhythmias. A percutaneous interventional approach was used to implant two Multilink stents (Advanced Cardiovascular Systems, Santa Clara, California, USA) of $3.0 \times 25 \mathrm{~mm}$ and $3.5 \times 15 \mathrm{~mm}$ in the bridging segment, and the systolic luminal narrowing disappeared (figs $1 \mathrm{C}$ and D). The stent delivery system tracked easily through the bridged area and both stents were deployed at 11 atm. No balloon predilatation was performed. After the procedure atenolol was withdrawn and the patient received antiplatelet treatment with aspirin and ticlopidine.

A second exercise thallium test performed five weeks later was normal at maximum theoretical heart rate. Control angiography at six months showed a mild minimum luminal diameter loss without restenosis (figs $1 \mathrm{E}$ and F). The patient remains free from angina and arrhythmias.

The incidence of myocardial bridging ranges from $5 \%$ to $85 \%$ in necropsy studies, while the reported angiographic prevalence is from $0.5 \%$ to $1.6 \%{ }^{1}$ The course is usually benign with a five year survival of $97.5 \%$; however, myocardial ischaemia, infarction, arrhythmias, and sudden death have been reported. ${ }^{2}$ 


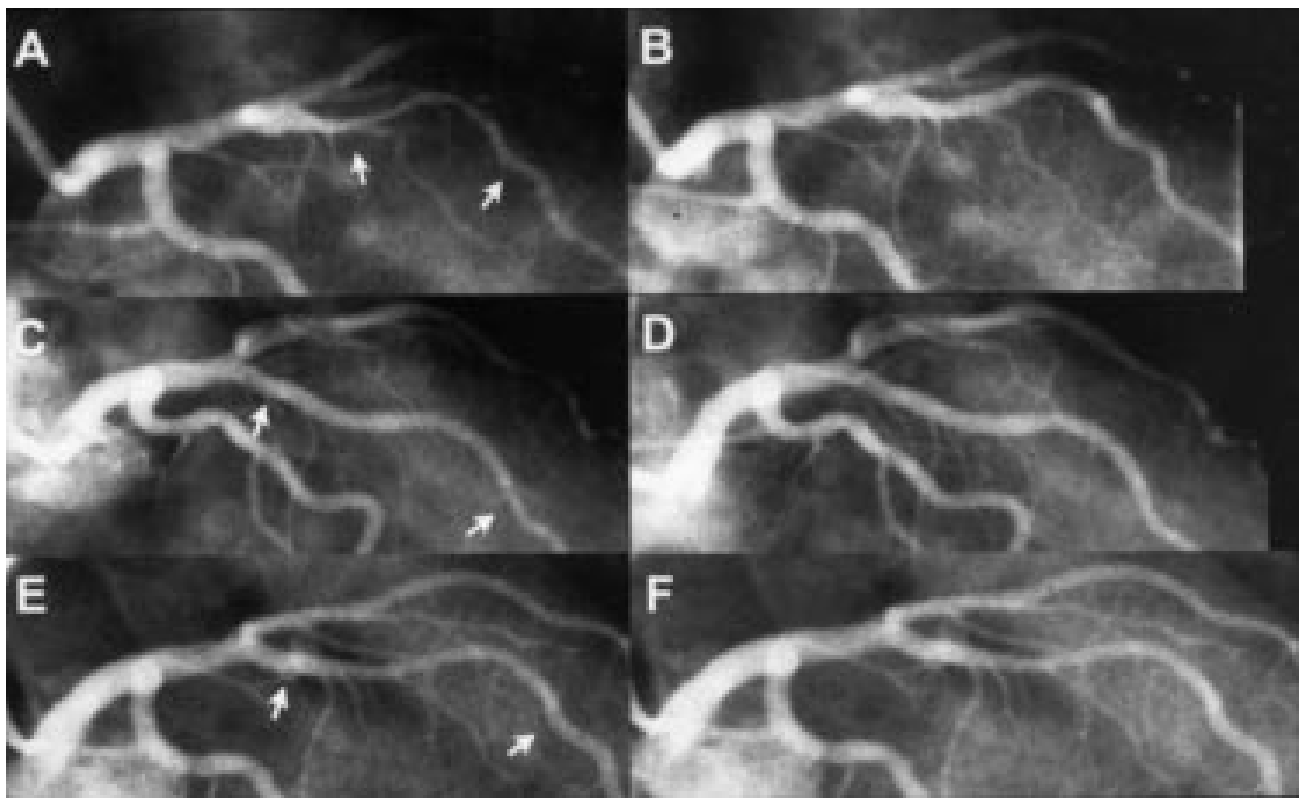

Figure 1 Angiographic changes observed in the bridged segment of the left anterior descending coronary artery (LAD) before and after coronary stenting and at six month' follow up. Milking effect of the medium segment of the LAD (arrows) during systole $(A)$, which disappeared during diastole (B). Angiograms in systole (C) and diastole (D) after the implantation of two consecutive Multilink stents (arrows). Selective left coronary angiograms in systole (E) and diastole (F) at six month' follow up.

To our knowledge, coronary stent implantation in myocardial bridging without angiographic evidence of arteriosclerotic lesions has been reported in only two patients who had acute myocardial infarction caused by the bridge. ${ }^{34}$ In both patients, a Palmaz-Schatz coronary stent (Johnson \& Johnson Interventional Systems, Warren, New Jersey, USA) was successfully implanted in the LAD. One patient was asymptomatic six months after the procedure, but no angiographic follow up was available. $^{3}$ In the other patient, angiography performed six weeks after the procedure for atypical chest pain did not show systolic compression at the stented segment. ${ }^{4}$ In our patient the systolic bridging of the LAD was associated with angina and possibly nonsustained monomorphic VT. Despite medical treatment the angina persisted; it stopped after the implantation of two coronary stents in the intramyocardial segment. Thallium-201 scintigraphy after stenting showed normalisation of myocardial distribution images. The stented bridging segment was longer than that previously described. ${ }^{34}$ The implantation of two coronary stents, covering a total length of $40 \mathrm{~mm}$ of coronary artery, was needed. We chose the Multilink stent because of its good trackability, flexibility, and resistance to exter- nal radial compression. ${ }^{5}$ This report is the first to show long term angiographic follow up after stenting of a myocardial bridge. No angiographic restenosis was observed six months after the procedure. However, coronary stent implantation may be ineffective in relieving angina in patients with widespread coronary bridging that involves the septal branches of the LAD artery. In addition, angina may recur because of luminal narrowing as a result of the proliferative response to endothelium damage during angioplasty, or because of shrinking of the scaffolding of the stent caused by external compression of the myocardial bridge.

1 Kramer JR, Kitazume H, Proudfit WL, et al. Clinical significance of isolated coronary bridges, benign and frequent condition involving the left anterior descending artery. $A m$ Heart f 1982;103:283-8.

2 Noble J, Bourassa MG, Petitclerc R, et al. Myocardial bridging and milking effect of the left anterior descending coronary artery: normal variant or obstruction? Am f Car-
diol 1976;37:993-9.

3 Stables RH, Knight CJ, McNeill JG, et al. Coronary stenting in the management of myocardial ischemia caused by muscle bridging. Br Heart 1995;74:90-2.

4 Smith SC, Taber MT, Robiolio PA, et al. Acute myocardial infarction caused by a myocardial bridge treated with intracoronary stenting. Cathet Cardiovas Diagn 1997;42:20912

5 Carter AJ, Laird JR, Kufs WM, et al. Coronary stenting with a novel stainless steel balloon-expandable stent: determinants of neointimal formation and changes in arterial geometry after placement in an atherosclerotic model. $\mathcal{F}$ 